\title{
Enhanced Production of Functional Proteins from Defective Genes
}

\author{
Biao Xi, Fangxia Guan, and David S. Lawrence* \\ Department of Biochemistry \\ The Albert Einstein College of Medicine \\ 1300 Morris Park Ave \\ Bronx, NY 10461
}

SUPPORTING INFORMATION

\section{EXPERIMENTAL PROCEDURES}

\section{Plasmid Construction}

The wild type human fibroblast cell line GM02037 (Coriell Cell Repositories, NJ) was used as the DNA template for atm promoter amplification. In brief, the Qiagen Blood \& Cell Culture DNA Mini kit was used to purify genomic DNA. ATM gene promoter primers, which were used to amplify the desired fragment DNA, were designed based on the sequence in Genebank. The forward primer sequence employed was GATCAAAACCACAGCAGGAAC and the reverse primer was GCCACGGGAGGAGGCGAG. PCR was carried out using the Roche Expand High Fidelity PCR system. The amplified atm promoter region was then cloned into the TOPO2.1 PCR vector (Invitrogen) for sequencing verification and subsequently subcloned into the promoterless plasmid PA3Luc. Firefly luciferase cDNA was mutated (QuikChange Site-Directed Mutagenesis Kit) in the desired region to furnish the three stop codons, as shown as Figure 2(c). The CMV driven Renilla luciferase plasmid was purchased from Promega as the internal control for the dual luciferase assay.

\section{Cell culture and transfection}

Human Kidney HEK293T cell was a gift from Professor Philipp Scherer in the Department of Cell Biology at the Albert Einstein College of Medicine. All other cell lines were purchased from Coriell Cell Repositories, NJ. HEK293T cells were maintained in Dulbecco's Modified Eagle Medium with $1 \mathrm{~g} / \mathrm{L}$ D-glucose and $10 \%$ fetal bovine serum. The Human AT fibroblast cell line GM05823 was cultured in MEM medium with $1 \mathrm{~g} / \mathrm{L}$ D-glucose and $15 \%$ fetal bovine serum. The Human lympoblastoid cell lines GM 13810 and GM13860 were cultured in RPMI 1640 medium with $10 \%$ heat inactivated fetal bovine serum and $1 \mathrm{~g} / \mathrm{L}$ of D-glucose. All cell lines were incubated at $37{ }^{\circ} \mathrm{C}$ in a humidified $5 \% \mathrm{CO}_{2}$ atmosphere. 
Transfections were performed using GeneJammer reagent (Stratagene) based on the protocol from the manufacturer. In brief, approximately $15 \times 10^{5}$ HEK293T cells were seeded into white 96 well plates (Corning) and grown for less than 24 hr. Cells were transfected with $0.6 \mu \mathrm{L}$ of GeneJammer and $0.1 \mu \mathrm{g}$ plasmids. In the dual luciferase assay, the plasmid ratio of ATM promoter-driven firefly luciferase to CMV promoter-driven Renilla luciferase was 30:1. In the dual luciferase experiments that utilized the stop codon-containing firefly luciferase genes, the ratio was 40:1. On the second day after transfection, the medium in each well was changed, and the promoter and/or nonsense-suppressing agents added to the wells for a $24 \mathrm{hr}$ incubation period. The cells were subsequently lysed and promoter activity/nonsense suppression efficiency then assessed via the dual luciferase assay.

\section{Dual luciferase assay}

The dual luciferase kit was purchased from Promega and the protocol employed was conducted according to the directions supplied by manufacturer. In brief, cells were washed with PBS and lysates were prepared using passive lysis buffer. Luminescence was determined with a Molecular Devices Lmax 96 well plate luminometer. Light emission was measured after each of the $100 \mu \mathrm{L}$. luminescence substrates and/or stop solutions were injected. ATM promoter activity was calculated by the ratio of relative light units of flash (firefly) to glow (renilla) luminescence in each well, which was compared with control wells (cultured only in medium with plasmid but no drug treatment) in each plate. In the case of the stop codon suppression studies, controls also included cells containing the plasmids without the inserted stop codon, which were treated with nonsense suppressing and/or promoter activating agents. All individual treatments in a given experiment were performed in triplicate and all experiments repeated 3 - 10 times.

A total of 8 drugs out of the $\sim 400$ screened upregulated the atm promoter 1.5 -fold or greater. The effect of ofloxacin and thioguanine concentration on atm promoter activity was examined. Ofloxacin: $2.5 \mu \mathrm{M}, 0.95 \pm 0.13$ fold; $25 \mu \mathrm{M}, 1.06 \pm 0.29$ fold; $250 \mu \mathrm{M}, 1.52 \pm 0.10$ fold; $750 \mu \mathrm{M}, 1.29 \pm 0.05$ fold. Thioguanine: $2.5 \mu \mathrm{M}$, $1.25 \pm 0.01$ fold; $5 \mu \mathrm{M}, 1.79 \pm 0.04$ fold; $25 \mu \mathrm{M}, 1.74 \pm 0.27$ fold; $50 \mu \mathrm{M}, 2.76 \pm$ 0.15 fold; $250 \mu \mathrm{M}, 1.56 \pm 0.02$ fold.

The effect of ofloxacin and thioguanine concentration on stop codon (opal) read through was examined where the gentamicin concentration employed was 300 $\mathrm{mg} / \mathrm{L}$. Ofloxacin: $5 \mu \mathrm{M}, 10.1 \pm 1.0$ fold; $50 \mu \mathrm{M}, 13.0 \pm 0.7$ fold; $150 \mu \mathrm{M}, 12.3 \pm 0.8$ fold; $250 \mu \mathrm{M}, 14.2 \pm 1.2$ fold; $500 \mu \mathrm{M}, 15.0 \pm 0.7$ fold; $1 \mathrm{mM}, 22.0 \pm 0.6$ fold. Thioguanine: $0.5 \mu \mathrm{M}, 10.8 \pm 0.7$ fold; $2.5 \mu \mathrm{M}, 12.5 \pm 1.2$ fold; $25 \mu \mathrm{M}, 39.7 \pm 2.0$ fold.

\section{Drug library and treatment}

Drugs were purchased from a variety of commercial sources and dissolved in appropriate solvents (DMSO, water, dioxane, or methanol) to furnish $10 \mathrm{mM}$ 
stock solutions ( $8 \times 12$ formatted stored in deep well plates). Diluted stocks ( 1 $\mathrm{mM}$ and $100 \mu \mathrm{M}$ in $0.1 \% \mathrm{BSA}, 1 \mathrm{mM}$ Tris, $\mathrm{pH} 7.5$ sterilized buffer) of the starting $10 \mathrm{mM}$ library were prepared using a Packard Multiprobe II HT EX liquid handling robot. These stock solutions were subsequently transferred into a series of $8 \times 12$ formatted shallow well plates to avoid exposure to repetitive freeze-thaw cycles. All plates were kept $-20^{\circ} \mathrm{C}$. The drug solutions were thawed at room temperature and used at the final concentrations of $2.5 \mu \mathrm{M}, 25 \mu \mathrm{M}$, and $250 \mu \mathrm{M}$. Before the drugs were employed to treat cells, the solutions in the individual wells were agitated using a multichannel pipettor. The drug solutions were subsequently added to cells cultured in wells of 96 well plates (where the final DMSO concentration is less than $2.5 \%$ ).

\section{mRNA Quantitation}

Single-stranded cDNAs were synthesized by reverse transcription, using a First Strand cDNA Synthesis kit (Invitrogen), and then used as a template in polymerase chain reactions. ATM mRNA was primarily quantified using real time PCR. In brief, cells were treated with the desired drugs or combinations thereof under conditions identical to those described above for the dual luciferase assay. Cells were lysed and RNA extracted with the TRIzol reagent (Invitrogen). Approximately $1 \mu \mathrm{g}$ of total RNA in each sample was subjected to cDNA synthesis using the First Strand cDNA Synthesis kit (Invitrogen). In the real time PCR reaction, the FAM labeled MGB probe, CCAGCTATTTGGTTTGAG, was designed by the Primer Express software from Applied Biosystems (AB) and synthesized by AB. The human $B$-actin MGB probes, VIC or FAM fluorescently labeled, were purchased from $A B$. All other reaction reagents were purchased from $A B$. PCR reactions were performed using the ABI PRISM 7000 Sequence Detection System in triplicate. Some samples were also tested on the ABI 7700 Sequence Detection System for verification and essentially identical results were obtained with both real time PCR instruments. All collected data were compared with a ß-actin control. In addition to real time PCR, the branched chain DNA (bDNA) RNA quantitation method was employed as well. Briefly, the QuantiGene kit as well as the customer designed atm probe sets were purchased from Genospectra. The latter probes target the same region on the atm message RNA region as the real time PCR probes. The cells were treated as described above for the dual luciferase assay. Cell lysates were transferred into a capture plate and atm mRNA allowed to hybridize with the probe set at $53{ }^{\circ} \mathrm{C}$ overnight. After 16-20 hr, the signal was amplified and subsequently read using a Molecular Devices Lmax luminometer. The internal human $ß$-actin probe sets were purchased from Genospectra. All experiments were performed in triplicate. 


\section{Library of FDA-Approved Drugs}

(unless otherwise indicated, drugs were stored as DMSO $10 \mathrm{mM}$ stock solutions and subsequently diluted as described above) 


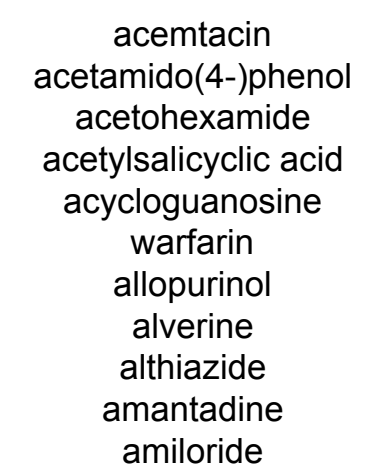

aminoglutethimide

amoxapine

aniracetam

antazoline

antipyrine

thioguanine

apomorphine hydrochloride

astemizole

atenolol

atropine

azlocilin

bendroflumethiazide

benzthiazide

bepridil

betahistine

betamethasone

bezafibrate

bretylium tosylate

budesonide

bumetanide

bupropion

busulfan

biphenylacetic acid

canrenoic acid

captopril

cefaclor

cefadroxil

chloramphenicol

chlormezanone

chloroquine

chlorpheniramine

ciclopirox olamine

cinnarizine

cinoxacin

cis-platinum(II)diamine

clemastine

clotrimazole

colchicine

cyproheptadine

cyclizine

chlorpropamide

\begin{tabular}{|c|c|c|}
\hline 53164-05-9 & RT & Sigma \\
\hline $103-90-2$ & RT & Sigma \\
\hline $968-81-0$ & RT & ICN \\
\hline $50-78-2$ & RT & Sigma \\
\hline $59277-89-3$ & RT & Sigma \\
\hline $81-81-2$ & RT & Sigma \\
\hline $315-30-0$ & RT & Sigma \\
\hline 128897-67-6 & RT & Sigma \\
\hline $5588-16-9$ & RT & Sigma \\
\hline $665-66-7$ & RT & Sigma \\
\hline 2016-88-8 & RT & Sigma \\
\hline $125-84-8$ & RT & Sigma \\
\hline $14028-44-5$ & RT & ICN \\
\hline $72432-10-1$ & RT & Sigma \\
\hline $154-68-7$ & RT & Sigma \\
\hline $60-80-0$ & RT & Sigma \\
\hline $154-42-7$ & RT & Sigma \\
\hline 41372-20-7 & RT & Aldrich \\
\hline 68844-77-9 & RT & Sigma \\
\hline 29122-68-7 & RT & Sigm \\
\hline $51-55-8$ & RT & Sigm \\
\hline $37091-66-0$ & RT & Sigm \\
\hline $73-48-3$ & RT & Sigm \\
\hline $91-33-8$ & RT & Sigme \\
\hline 74764-40-2 & RT & Sigma \\
\hline 5579-84-0 & RT & Sigma \\
\hline $378-44-9$ & RT & Sigm \\
\hline $41859-67-0$ & RT & Sigm \\
\hline $61-75-6$ & RT & Sigm \\
\hline 51333-22-3 & RT & Sigm \\
\hline 28395-03-1 & RT & Sigma \\
\hline 31677-93-7 & RT & ICI \\
\hline $55-98-1$ & RT & Sigma \\
\hline $5728-52-9$ & RT & Alfá \\
\hline 2181.04 .6 & RT & Sigma \\
\hline 62571-86-2 & RT & Sigma \\
\hline 70356-03-5 & RT & Sigma \\
\hline 66592-87-8 & RT & Sigma \\
\hline $56-75-7$ & RT & Sigma \\
\hline $80-77-3$ & RT & ICN \\
\hline $50-63-5$ & RT & Sigma \\
\hline $2438-32-6$ & RT & Sigma \\
\hline $41621-49-2$ & RT & Sigma \\
\hline $298-57-7$ & RT & Sigma \\
\hline 28657-80-9 & RT & Sigma \\
\hline $15663-271-1$ & RT & Sigma \\
\hline 14976-57-9 & RT & Sigma \\
\hline 23593-75-1 & RT & Sigma \\
\hline $64-86-8$ & RT & Sigma \\
\hline $969-33-5$ & RT & Sigma \\
\hline $303-25-3$ & RT & $\mathrm{ICI}$ \\
\hline $94-20-2$ & RT & Sigm \\
\hline
\end{tabular}




\author{
clomipramine \\ clozapine \\ debrisoquine sulfate \\ deprenyl hydrochloride \\ floxuridine \\ diazoxide \\ diclofenac \\ dicyclomine \\ diethylstilbestrol \\ diflunisal \\ digitoxin \\ digoxin \\ dihydroergotamine \\ dimenhydrinate \\ dimethyl $(1,1)$ biguanide \\ diphenhydramine \\ DOPA \\ disopyramide \\ phenytoin \\ doxepine \\ doxylamine \\ domperidone \\ econazole \\ erythromycin \\ eserine \\ ethacrynic acid \\ ethambutol \\ ethyl p-aminobenzoate \\ etoposide \\ mestranol \\ estradiol \\ famotidine \\ fenbufen \\ fenofibrate \\ fenoprofen \\ fenoterol \\ flavoxate \\ fluconazole \\ flunisolide \\ fluoro(5-)uracil \\ flupenthixol-cis \\ fluphenazine \\ flurbiprofen \\ flutamide \\ furosemide \\ flucytosine \\ gemfibrozil \\ guanethidine \\ haloperidol \\ hexamethylenetetramine \\ hydralazine \\ hydrochlorothiazide \\ hydrocortisone \\ hydroflumethiazide
}

$\begin{array}{ccr}303-49-1 & \text { RT } & \text { Sigma } \\ 5786-21-0 & \text { RT } & \text { Sigma } \\ 581-88-4 & \text { RT } & \text { Sigma } \\ 14611-52-0 & \text { RT } & \text { ICN } \\ 50-91-9 & \text { RT } & \text { TCI } \\ 364-98-7 & \text { RT } & \text { Sigma } \\ 15307-79-6 & \text { RT } & \text { Sigma } \\ 67-92-5 & \text { RT } & \text { Sigma } \\ 56-53-1 & \text { RT } & \text { Sigma } \\ 22494-42-4 & \text { RT } & \text { Sigma } \\ 71-63-6 & \text { RT } & \text { Sigma } \\ 20830-75-5 & \text { RT } & \text { Sigma } \\ 6190-39-2 & \text { RT } & \text { Sigma } \\ 523-87-5 & \text { RT } & \text { Sigma } \\ 15537-72-1 & \text { RT } & \text { Sigma } \\ 147-24-0 & \text { RT } & \text { Sigma } \\ 59-92-7 & \text { RT } & \text { Sigma } \\ 22059-60-5 & \text { RT } & \text { Sigma } \\ 57-41-0 & \text { RT } & \text { Sigma } \\ 1229-29-4 & \text { RT } & \text { Sigma } \\ 469-21-6 & \text { RT } & \text { Sigma } \\ 57808-66-9 & \text { RT } & \text { ICN } \\ 24169-02-6 & \text { RT } & \text { Sigma } \\ 114-07-8 & \text { RT } & \text { Sigma } \\ 57-64-7 & \text { RT } & \text { Sigma } \\ 58-54-8 & \text { RT } & \text { Sigma } \\ 1070-11-7 & \text { RT } & \text { Sigma } \\ 94-09-7 & \text { RT } & \text { Sigma } \\ 33419-42-0 & \text { RT } & \text { Sigma } \\ 72-33-3 & \text { RT } & \text { Aldrich } \\ 50-28-2 & \text { RT } & \text { Sigma } \\ 76824-35-6 & \text { RT } & \text { ICN } \\ 36330-85-5 & \text { RT } & \text { Sigma } \\ 49562-28-9 & \text { RT } & \text { Sigma } \\ 53746-45-5 & \text { RT } & \text { Sigma } \\ 1944.12 .3 & \text { RT } & \text { Sigma } \\ 3717-88-2 & \text { RT } & \text { ICN } \\ 86386-73-4 & \text { RT } & \text { ICN } \\ 77326-96-6 & \text { RT } & \text { Sigma } \\ 51-21-8 & \text { RT } & \text { Sigma } \\ 2709-56-0 & \text { RT } & \text { ICN } \\ 146-56-5 & \text { RT } & \text { Sigma } \\ 5104-49-4 & \text { RT } & \text { Sigma } \\ 13311-84-7 & \text { RT } & \text { Sigma } \\ 54-31-9 & \text { RT } & \text { Sigma } \\ 2022-85-7 & \text { RT } & \text { TCI } \\ 25812-30-0 & \text { RT } & \text { Sigma } \\ 645-43-2 & \text { RT } & \text { Sigma } \\ 52-86-8 & \text { RT } & \text { Sigma } \\ 587-23-5 & \text { RT } & \text { Sigma } \\ 304-20-1 & \text { RT } & \text { Sigma } \\ 58-93-5 & \text { RT } & \text { Sigma } \\ 50-23-7 & \text { RT } & \text { Sigma } \\ 135-09-1 & \text { RT } & \text { Sigma } \\ & & \end{array}$




\begin{tabular}{|c|c|c|c|c|c|c|}
\hline 2 & $B$ & 5 & hydroxyzinc & $10246-75-0$ & $\mathrm{RT}$ & Sigma \\
\hline 2 & B & 6 & ibuprofen & $15687-27-1$ & RT & Sigma \\
\hline 2 & B & 7 & imipramine & $713-52-0$ & RT & ICN \\
\hline 2 & $\mathrm{~B}$ & 8 & indapamide & $26807-65-8$ & $\mathrm{RT}$ & Sigma \\
\hline 2 & B & 9 & indomethicin & $53-86-1$ & $\mathrm{RT}$ & Sigma \\
\hline 2 & $\mathrm{~B}$ & 10 & ipratropium bromide & $22254-24-6$ & RT & Sigma \\
\hline 2 & $\mathrm{~B}$ & 11 & isonicotinic acid hydrazide & $54-85-3$ & $\mathrm{RT}$ & Sigma \\
\hline 2 & $\mathrm{~B}$ & 12 & isosorbide dinitrate & $87-33-2$ & $\mathrm{RT}$ & Sigma \\
\hline 2 & C & 1 & kanamycin sulfate & 25389-94-0 & $\mathrm{RT}$ & Sigma \\
\hline 2 & $\mathrm{C}$ & 2 & ketoprofen & $22071-15-4$ & $\mathrm{RT}$ & Sigma \\
\hline 2 & C & 3 & ketotifen & $34580-14-8$ & $\mathrm{RT}$ & Sigma \\
\hline 2 & C & 4 & ketoconazole & $65277-42-1$ & $\mathrm{RT}$ & $\mathrm{ICN}$ \\
\hline 2 & $\mathrm{C}$ & 5 & isoproterenol-(-) & $54750-10-6$ & $\mathrm{RT}$ & Sigma \\
\hline 2 & C & 6 & labetalol & $32780-64-6$ & $\mathrm{RT}$ & Sigma \\
\hline 2 & C & 7 & lidocaine & $6108-05-0$ & $\mathrm{RT}$ & Sigma \\
\hline 2 & C & 8 & lithium carbonate & $554-13-2$ & $\mathrm{RT}$ & Sigma \\
\hline 2 & $\mathrm{C}$ & 9 & loperamide & $34552-83-5$ & $\mathrm{RT}$ & Sigma \\
\hline 2 & C & 10 & magnesium sulfate & $10034-99-8$ & RT & Sigma \\
\hline 2 & C & 11 & mannitol-(D) & 69-65-8 & $\mathrm{RT}$ & Sigma \\
\hline 2 & C & 12 & maprotiline & $10262-69-8$ & $\mathrm{RT}$ & Sigma \\
\hline 2 & D & 1 & meclofenamic acid & $36385-02-0$ & RT & Sigma \\
\hline 2 & D & 2 & mefenamic acid & $61-68-7$ & $\mathrm{RT}$ & Sigma \\
\hline 2 & $\mathrm{D}$ & 3 & $\begin{array}{c}\text { acetoxy(17 alpha)-6-methyl-4,6-pregnadiene- } \\
\text { 3,20-dione }\end{array}$ & $595-33-5$ & RT & Sigma \\
\hline 2 & D & 4 & metaproterenol & $5874-97-5$ & $\mathrm{RT}$ & Sigma \\
\hline 2 & D & 5 & metoclopramide & $7232-21-5$ & $\mathrm{RT}$ & Sigma \\
\hline 2 & $\mathrm{D}$ & 6 & metolazone & $17560-51-9$ & $\mathrm{RT}$ & $\mathrm{ICN}$ \\
\hline 2 & $\mathrm{D}$ & 7 & metoprolol & $56392-17-7$ & $\mathrm{RT}$ & Sigma \\
\hline 2 & D & 8 & metricane & $1084-65-7$ & $\mathrm{RT}$ & Sigma \\
\hline 2 & $\mathrm{D}$ & 9 & melphalan & $148-82-3$ & $\mathrm{RT}$ & Sigma \\
\hline 2 & $\mathrm{D}$ & 10 & mercapto(6-)purine & $6112-76-1$ & $\mathrm{RT}$ & Sigma \\
\hline 2 & D & 11 & methyl(6alpha)-17-alpha-hydroxyprogesterone & $520-85-4$ & $\mathrm{RT}$ & Sigma \\
\hline 2 & $\mathrm{D}$ & 12 & medrol & $83-43-2$ & $\mathrm{RT}$ & Sigma \\
\hline 2 & $E$ & 1 & miconazole & $75319-48-1$ & $\mathrm{RT}$ & Sigma \\
\hline 2 & $E$ & 2 & minoxidil & $38304-91-5$ & $\mathrm{RT}$ & Sigma \\
\hline 2 & $E$ & 3 & mitoxantrone & $65271-80-9$ & RT & Sigma \\
\hline 2 & $E$ & 4 & nabumetone & $42924-53-8$ & RT & Sigma \\
\hline 2 & $E$ & 5 & nadolol & $42200-33-9$ & $\mathrm{RT}$ & Sigma \\
\hline 2 & $E$ & 6 & naphazoline hydrochloride & $550-99-2$ & RT & Sigma \\
\hline 2 & $E$ & 7 & naproxen & 26159-34-2 & $\mathrm{RT}$ & Sigma \\
\hline 2 & $E$ & 8 & nefopam & $23327-57-3$ & $\mathrm{RT}$ & ICN \\
\hline 2 & $E$ & 9 & neomycin sulfate & $1405-10-3$ & RT & Sigma \\
\hline 2 & $E$ & 10 & nicotine-(-) solution & $65-30-5$ & $\mathrm{RT}$ & Sigma \\
\hline 2 & $E$ & 11 & nimodipine & $66085-59-4$ & RT & ICN \\
\hline 2 & $E$ & 12 & nitrendipine & $39562-70-4$ & RT & ICN \\
\hline 2 & $\mathrm{~F}$ & 1 & nitrofurantoin & $67-20-9$ & $\mathrm{RT}$ & Sigma \\
\hline 2 & $\mathrm{~F}$ & 2 & nortriptyline & $894-71-3$ & $\mathrm{RT}$ & Sigma \\
\hline 2 & $F$ & 3 & oxybutynin chloride & $1508-65-2$ & RT & Sigma \\
\hline 2 & $\mathrm{~F}$ & 4 & oxymetazoline hydrochloride & $2315-028$ & RT & Sigma \\
\hline 2 & $\mathrm{~F}$ & 5 & oxytetracycline & $79-57-2$ & RT & Sigma \\
\hline 2 & $\mathrm{~F}$ & 6 & penicillin G & 6130-64-0 & RT & Sigma \\
\hline 2 & $\mathrm{~F}$ & 7 & phenelzinc & $156-51-4$ & RT & Sigma \\
\hline 2 & $\mathrm{~F}$ & 8 & pheniramine & $86-21-5$ & RT & Sigma \\
\hline 2 & $\mathrm{~F}$ & 9 & phenoxymethyl penicillinic acid & $87-08-1$ & $\mathrm{RT}$ & Sigma \\
\hline
\end{tabular}




$\begin{array}{ccc}2 & \mathrm{~F} & 10 \\ 2 & \mathrm{~F} & 11 \\ 2 & \mathrm{~F} & 12 \\ 2 & \mathrm{G} & 1 \\ 2 & \mathrm{G} & 2 \\ 2 & \mathrm{G} & 3 \\ 2 & \mathrm{G} & 4 \\ 2 & \mathrm{G} & 5 \\ 2 & \mathrm{G} & 6 \\ 2 & \mathrm{G} & 7 \\ 2 & \mathrm{G} & 8 \\ 2 & \mathrm{G} & 9 \\ 2 & \mathrm{G} & 10 \\ 2 & \mathrm{G} & 11 \\ 2 & \mathrm{G} & 12 \\ 2 & \mathrm{H} & 1 \\ 2 & \mathrm{H} & 2 \\ 2 & \mathrm{H} & 3 \\ 2 & \mathrm{H} & 4 \\ 2 & \mathrm{H} & 5 \\ 2 & \mathrm{H} & 6 \\ 3 & \mathrm{~A} & 1 \\ 3 & \mathrm{~A} & 2 \\ 3 & \mathrm{~A} & 3 \\ 3 & \mathrm{~A} & 4 \\ 3 & \mathrm{~A} & 5 \\ 3 & \mathrm{~A} & 6 \\ 3 & \mathrm{~A} & 7 \\ 3 & \mathrm{~A} & 8 \\ 3 & \mathrm{~A} & 9 \\ 3 & \mathrm{~A} & 10 \\ 3 & \mathrm{~A} & 11 \\ 3 & \mathrm{~A} & 12 \\ 3 & \mathrm{~B} & 1 \\ 3 & \mathrm{~B} & 2 \\ 3 & \mathrm{~B} & 3 \\ 3 & \mathrm{C} & 6 \\ 3 & \mathrm{C} & 7 \\ 3 & \mathrm{C} & 8 \\ 3 & \mathrm{~B} & 4 \\ 3 & \mathrm{~B} & 6 \\ 3 & \mathrm{~B} & 7 \\ 3 & \mathrm{~B} & 8 \\ 3 & \mathrm{~B} & 9 \\ 3 & \mathrm{~B} & 10 \\ 3 & \mathrm{~B} & 11 \\ 3 & \mathrm{~B} & 12 \\ 3 & \mathrm{C} & 1 \\ 3 & \mathrm{C} & 2 \\ 3 & \mathrm{C} & 3 \\ & & \\ 3 & \mathrm{C} & 5 \\ 3\end{array}$

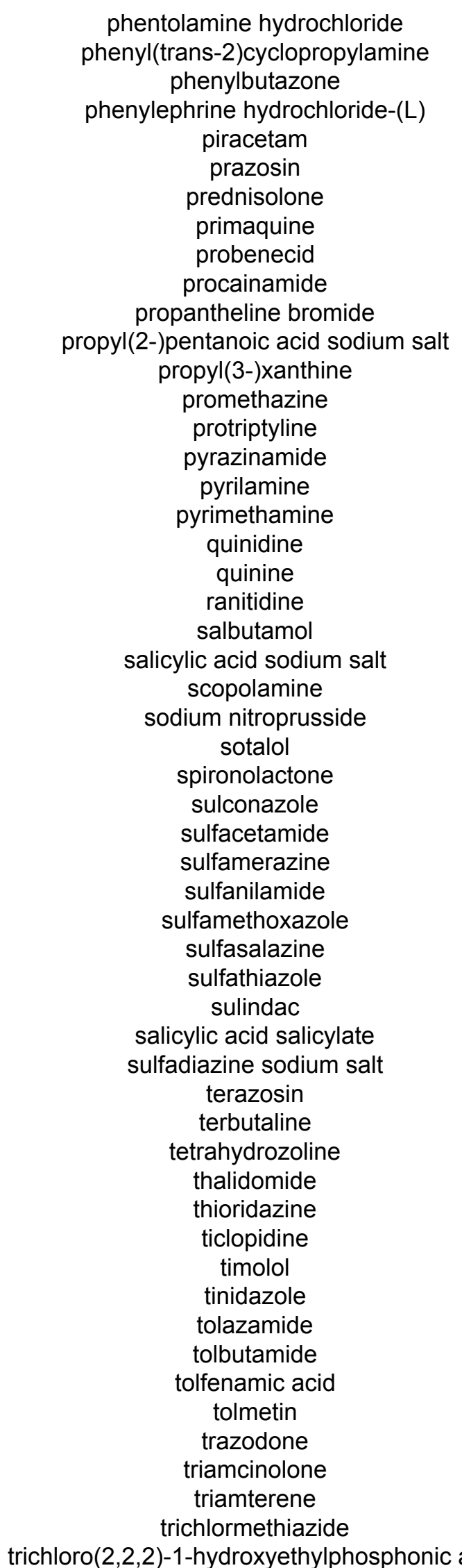

phentolamine hydrochloride phenylbutazone

\begin{tabular}{|c|c|c|}
\hline $73-05-2$ & $\mathrm{RT}$ & Sigma \\
\hline $95-62-5$ & RT & ICN \\
\hline $50-33-9$ & RT & Sigma \\
\hline $61-76-7$ & $\mathrm{RT}$ & Sigma \\
\hline $7491-74-9$ & $\mathrm{RT}$ & Sigma \\
\hline $19237-84-4$ & $\mathrm{RT}$ & Sigma \\
\hline $50-24-8$ & $\mathrm{RT}$ & Sigma \\
\hline $63-45-6$ & $\mathrm{RT}$ & ICN \\
\hline $57-66-9$ & $\mathrm{RT}$ & Sigma \\
\hline 614-39-1 & $\mathrm{RT}$ & Sigma \\
\hline $50-34-0$ & $\mathrm{RT}$ & Sigma \\
\hline $1069-66-5$ & $\mathrm{RT}$ & Sigma \\
\hline $41078-02-8$ & RT & Sigma \\
\hline $58-33-3$ & $\mathrm{RT}$ & Sigma \\
\hline $1225-55-4$ & $\mathrm{RT}$ & Sigma \\
\hline $98-96-4$ & RT & Sigma \\
\hline $91-84-9$ & RT & Sigma \\
\hline $58-14-0$ & $\mathrm{RT}$ & Sigma \\
\hline $6151-40-2$ & RT & Sigma \\
\hline $6119-47-7$ & RT & Sigma \\
\hline $66357-35-5$ & RT & $\mathrm{ICN}$ \\
\hline $36519-31-0$ & RT & Sigma \\
\hline $54-21-7$ & RT & Sigma \\
\hline $114-49-87$ & RT & Sigma \\
\hline $13755-38-9$ & RT & ICN \\
\hline $959-24-0$ & RT & Sigma \\
\hline $52-01-7$ & RT & Sigma \\
\hline $61318-91-0$ & RT & Sigma \\
\hline $144-80-9$ & RT & Sigma \\
\hline $127-79-7$ & RT & Sigma \\
\hline $63-74-1$ & RT & Sigma \\
\hline $723-46-6$ & RT & Sigma \\
\hline $599-79-1$ & RT & Sigma \\
\hline $72-14-0$ & RT & $\mathrm{ICN}$ \\
\hline 38194-50-2 & RT & Sigma \\
\hline $552-94-3$ & RT & $\mathrm{TCl}$ \\
\hline $68-35-9$ & RT & Sigma \\
\hline 63590-64-7 & RT & Sigma \\
\hline $23031-32-5$ & RT & Sigma \\
\hline $522-48-5$ & RT & Sigma \\
\hline $50-35-1$ & RT & $\mathrm{ICN}$ \\
\hline $130-61-0$ & RT & Sigma \\
\hline $53885-35-1$ & RT & Sigma \\
\hline $26921-17-5$ & RT & Sigma \\
\hline $19387-91-8$ & RT & Sigma \\
\hline $1156-19-0$ & RT & Sigma \\
\hline $64-77-7$ & RT & Sigma \\
\hline $13710-19-5$ & RT & Sigma \\
\hline 64490-92-2 & RT & Sigma \\
\hline 25332-39-2 & RT & Sigma \\
\hline $124-94-7$ & RT & Sigma \\
\hline $396-01-0$ & RT & Sigma \\
\hline $133-67-5$ & RT & Sigma \\
\hline $52-68-6$ & RT & Sigm \\
\hline
\end{tabular}


3 C 10

$\begin{array}{lll}3 & C & 11\end{array}$

$\begin{array}{lll}3 & \text { C } & 12\end{array}$

3 D 1

3 D 2

3 D 3

3 D 4

3 D 5

4 A 1

4 A 2

4 A 3

4 A 4

4 A 5

4 A 6

4 A 7

4 A 8

4 A 9

4 A 10

4 A 11

4 A 12

4 B 1

4 B 2

4 B 3

4 B 4

4 B 5

4 B 6

4 B 7

4 B 8

4 B 9

4 B 10

4 B 11

$\begin{array}{lll}4 & \text { B } & 12\end{array}$

$\begin{array}{lll}4 & \text { C } & 1\end{array}$

4 C 2

4 C 3

4 C 4

4 C 5

4 C 6

$\begin{array}{lll}4 & \text { C } 7\end{array}$

5 A 1

5 A 2

5 A 3

5 A 4

5 A 5

5 A 6

5 A 7

5 A 8

5 A 9

5 A 10

5 A 11

$\begin{array}{lll}5 & \text { A } 12\end{array}$

$\begin{array}{lll}5 & \text { B } & 1\end{array}$

5 B 2 dimethyl ester

trihexylphenidyl

trimeprazine

trimethobenzamide

verapamil

xylometazoline zopiclone

dextromethorphan primidone

amphetamine(D) sulfate butalbital

cannabidol

cannabinol

clonazepam codeine

desmethyldiazepam diazepam

fenluramine hydrochloride flunitrazepam

hydromorphone hydrochloride

meperidine hydrochloride meprobamate

mescaline hydrochloride

methadone hydrochloride

methamphetamine $(+)$ hydrochloride

nalorphine hydrochloride

pentobarbital

phenobarbital

temazepam

secobarbitol

tetrahydrocannabinol (delta 8)

tetrahydrocannabinol (delta 9)

cyclophosphoramide

amylobarbitone

vincristine

daunorubicin

galanthamine

vinblastine

mitomycin $\mathrm{C}$

actinomycin $D$

aminophylline

azathioprine

capreomycin

carmustine

chlortetracycline

cycloserine-(D)

cycloserine-(L)

demeclocycline

diphenylpyraline

dipyridamole

etridronic acid disodium salt griseofulvin

guanabenz

L-3,4-dihydroxyphenylalanine methyl ester

\begin{tabular}{|c|c|c|}
\hline $8947-95-8$ & RT & Yli \\
\hline 4330-99-8 & RT & Sigm \\
\hline $554-92-7$ & RT & in \\
\hline $23313-68-0$ & RT & Sigm \\
\hline $1218-35-3$ & RT & \\
\hline $43200-80-2$ & RT & \\
\hline $6700-$ & RT & \\
\hline 125 & RT & \\
\hline $60-$ & $\mathrm{MeOH}$ soln & \\
\hline $77-26-9$ & $\mathrm{MeOH}$ soln & \\
\hline $29-1$ & $\mathrm{MeOH}$ soln & \\
\hline 521 & $\mathrm{MeOH}$ & \\
\hline 1622 & $\mathrm{MeOH}$ soln & \\
\hline & $\mathrm{MeOH}$ & \\
\hline 1088 & $\mathrm{MeOH}$ & \\
\hline 439 & $\mathrm{MeOH}$ & \\
\hline 3616 & $\mathrm{MeC}$ & \\
\hline 1622 & $\mathrm{MeC}$ & \\
\hline $71-$ & $\mathrm{Me}$ & \\
\hline 50 & $\mathrm{Me}$ & \\
\hline 57 & $\mathrm{Me}$ & \\
\hline $54-$ & $\mathrm{Me}$ & \\
\hline 1095 & $\mathrm{Me}$ & \\
\hline & $\mathrm{Me}$ & \\
\hline 62 & $\mathrm{Me}$ & \\
\hline & $\mathrm{Me}$ & \\
\hline & $\mathrm{Me}$ & \\
\hline 846 & $\mathrm{Me}$ & \\
\hline \multirow[t]{3}{*}{$309-43-3$} & $\mathrm{Me}$ & \\
\hline & & \\
\hline & & \\
\hline-2 & & 0 \\
\hline & & \\
\hline & & \\
\hline $0-6$ & & \\
\hline & & ci \\
\hline & $\mathrm{MeC}$ & Sigr \\
\hline & & Sia \\
\hline & $\mathrm{H}_{2} \mathrm{O}$ & igm \\
\hline & & igm \\
\hline & & \\
\hline & & igm \\
\hline & & Sigm \\
\hline & & igm \\
\hline & & o: \\
\hline $339-72-0$ & eeze & Sigm \\
\hline & eeze & Sigm \\
\hline 132 & eeze & \\
\hline \multirow[t]{2}{*}{$58-32-2$} & eeze & 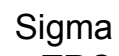 \\
\hline & & \\
\hline & & \\
\hline 505 & freeze & \\
\hline $142-0 v^{\prime}$ & freeze & \\
\hline
\end{tabular}


amino(9)-1,2,3,4-tetrahydroacridine

lanatoside $\mathrm{C}$

methotrexate

nalidixic acid

nystatin

pergolide

rifampicin

tetracycline

vitamin A

acebutolol

amikacin sulfate

amiodarone

amitriptyline

amoxicilin

amphotericin B

ampicilin trihydrate

amrinone

aztreonam

beclomethasone

benzydamine

benztropine

bromo(2)-alpha-ergocryptine

buspirone

carbamazepine

carbamyl-beta-methylcholine

carbenicillin

cefmetazole

cefoperazone

cefotaxime

ceftriaxone

cefuroxime

cephalothin

cephradine

chlorambucil

chlorothiazide

chlorprothixene

chlorthalidone

clofibrate

cimetidine

ciprofibrate

ciprofloxacin

clonidine

clopamide

cloxacillin

colistin

cyproterone acetate

cytosine beta-D-arabinofuranoside

dacarbazine

desipramine

dexamethasone

dicloxacillin

dihydrotachysterol

dobutamine

doxycycline

\begin{tabular}{|c|c|c|}
\hline & 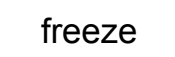 & 7 \\
\hline 2 & & . \\
\hline & & 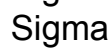 \\
\hline & & gma \\
\hline 1400 & & $\mathrm{gm}$ \\
\hline 66104 & & igm \\
\hline 329 & & igm \\
\hline $60-54-8$ & & Sigm \\
\hline $302-79-4$ & & Sigm \\
\hline $8-5$ & & Sigm \\
\hline $5-5$ & & Sign \\
\hline $5-3$ & & . \\
\hline 549 & & igm \\
\hline $8-0$ & & Sigm \\
\hline $9-3$ & & 0 \\
\hline-2 & & 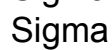 \\
\hline 4-8 & & $\because$ \\
\hline 8-0 & & IC \\
\hline & & iin \\
\hline & & sigm \\
\hline & & \\
\hline-1 & & \\
\hline & & \\
\hline & & \\
\hline & & \\
\hline & & \\
\hline & & \\
\hline & & \\
\hline & & \\
\hline & & \\
\hline & & ( \\
\hline & & \\
\hline & & $\mathrm{S}$ \\
\hline & & S \\
\hline & & Sigm \\
\hline & & Sigm \\
\hline & & Sig \\
\hline & & Sigm \\
\hline & & Sigm \\
\hline & & Sigm \\
\hline & & $\mathrm{IC}$ \\
\hline & & Sigm \\
\hline & & Sigm \\
\hline & & Sigma \\
\hline & & Sigma \\
\hline & & Sigm \\
\hline & & Sigm \\
\hline 434 & & Sigma \\
\hline & & Sigm \\
\hline & & Sigma \\
\hline 311 & & Sigm \\
\hline & & \\
\hline 49745 & & \\
\hline $17086-28$ & & \\
\hline
\end{tabular}


droperidol

enoxacin

epinephrine

ergocalciferol

ethionamde

flecainide

flunarizine

fusidic acid

glibenclamide

hydroxyurea

isoetharine

ivermectin

lansoprazole

lincomycin

hydroxychloroquine sulfate

mebeverine

meclizine

methoxamine hydrochloride

methyl(8-)-N-vanillyl-6-nonenamide

metronidazole

mexiletine

mianserin

naloxone

nicardipine

nicergoline

nifedipine

norfloxacin

novobiocin

ofloxacin

orphenadrine

oxacillin

penicillamine-(D)

penicillamine-(L)

perphanazine

phenindione

phenoxybenzamine

phosphomycin

pimozide

pindolol

piperacillin sodium salt

piroxicam

prochlorperazine

procyclidine

promazine

propafenone

propericyazine

propranolol

rescinnamine

ribavirin

streptomycin sulfate

sulfabenzamide

sulfamethizole

sulpriride

tamoxifen citrate

\begin{tabular}{|c|c|c|}
\hline & & \\
\hline 294-96-2 & rate & $\mathrm{gm}$ \\
\hline $2-3$ & & \\
\hline & & S \\
\hline $536-33-4$ & & s \\
\hline $4143-56-5$ & & \\
\hline $77-6$ & & \\
\hline 6.3 & & sig \\
\hline 10238 & & \\
\hline 127. & & \\
\hline 7279 & & S \\
\hline 36-7 & & \\
\hline $45-3$ & & S \\
\hline 154 & & $\mathrm{Sic}$ \\
\hline 747 & & $\mathrm{Acl}$ \\
\hline 275 & & Sig \\
\hline $2-9$ & & Sigr \\
\hline & & \\
\hline 404 & & Sign \\
\hline 443 & & DIg \\
\hline $1-4$ & & $\lg$ \\
\hline $7-7$ & & $\mathrm{~s} / \mathrm{r}$ \\
\hline $0-8$ & & sign \\
\hline $4-3$ & & sign \\
\hline $4-6$ & & إlg \\
\hline $5-4$ & & sign \\
\hline $6-7$ & & $\operatorname{sig}$ \\
\hline & & \\
\hline $6-1$ & & Sic \\
\hline & & Sigm \\
\hline & & \\
\hline 22 & & $\varsigma$ \\
\hline 11 & & \\
\hline & & \\
\hline & & Sig \\
\hline & & \\
\hline & & \\
\hline & & \\
\hline & & \\
\hline & & \\
\hline & & \\
\hline & & \\
\hline & & \\
\hline & & \\
\hline $56-5$ & & $S$ \\
\hline & & \\
\hline $9-0$ & & S \\
\hline $4-5$ & & \\
\hline C & & \\
\hline & & \\
\hline & & \\
\hline & & \\
\hline & & \\
\hline & & \\
\hline
\end{tabular}




$\begin{array}{llc}6 & \text { C } & 9 \\ 6 & \text { C } & 10 \\ 6 & \text { C } & 11 \\ 6 & \text { C } & 12 \\ 6 & \text { D } & 1 \\ 6 & \text { D } & 2 \\ 6 & \text { D } & 3 \\ 6 & \text { D } & 4 \\ 6 & \text { D } & 5 \\ 6 & \text { D } & 6 \\ 6 & \text { D } & 7 \\ 6 & \text { D } & 8 \\ 6 & \text { D } & 9 \\ 6 & \text { D } & 10 \\ 6 & \text { D } & 11 \\ 6 & \text { D } & 12 \\ 6 & \text { E } & 1 \\ 6 & \text { E } & 2 \\ 6 & \text { E } & 3 \\ 6 & \text { E } & 4 \\ 6 & \text { E } & 5\end{array}$

terfenadine
thiothixene
tobramycin sulfate
tolnaftate
trimethoprim
trimipramine
tripolidine
tulobuterol
vancomycin
diltiazem
milrinone
cyclosporin A
aclarubicin
pargyline
noscapine hydrochloride
choro(-2)deoxyadenosine
clindamycin
fluoro(2)adenine 9-beta-D-arabinofuranoside
netilmicin
doxorubicin
oxiracetam

$\begin{array}{ccc}50679-08-8 & \text { refrigerate } & \text { Sigma } \\ 5591-45-7 & \text { refrigerate } & \text { Sigma } \\ 79645-27-5 & \text { refrigerate } & \text { Sigma } \\ 2398-96-1 & \text { refrigerate } & \text { Sigma } \\ 738-70-5 & \text { refrigerate } & \text { Sigma } \\ 521-78-8 & \text { refrigerate } & \text { Sigma } \\ 6138-79-0 & \text { refrigerate } & \text { Sigma } \\ 56776-01-3 & \text { refrigerate } & \text { Sigma } \\ 1404-90-6 & \text { refrigerate } & \text { Sigma } \\ 33286-22-5 & \text { refrigerate } & \text { Sigma } \\ 78415-72-2 & \text { refrigerate } & \text { Sigma } \\ 59865-13-3 & \text { refrigerate } & \text { Sigma } \\ 75443-99-1 & \text { refrigerate } & \text { Sigma } \\ \text { P8010 } & \text { refrigerate } & \text { Sigma } \\ 912-60-6 & \text { refrigerate } & \text { Sigma } \\ 429-63-8 & \text { refrigerate } & \text { Sigma } \\ 21462-39-5 & \text { refrigerate } & \text { Sigma } \\ 21679-14-1 & \text { refrigerate } & \text { Sigma } \\ 56391-57-2 & \text { refrigerate } & \text { Sigma } \\ 25316-40-9 & \text { refrigerate } & \text { Sigma } \\ 62613-82-5 & \text { refrigerate } & \text { Sigma }\end{array}$

